\title{
Boundedness for Commutators of Bilinear $\theta$-Type Calderón-Zygmund Operators on Nonhomogeneous Metric Measure Spaces
}

\author{
Rulong Xie, ${ }^{1,2}$ Lisheng Shu, ${ }^{3}$ and Aiwen Sun ${ }^{3}$ \\ ${ }^{1}$ Department of Mathematics, Chaohu University, Hefei 238000, China \\ ${ }^{2}$ School of Mathematical Sciences, University of Science and Technology of China, Hefei 230026, China \\ ${ }^{3}$ Department of Mathematics, Anhui Normal University, Wuhu 241000, China \\ Correspondence should be addressed to Lisheng Shu; shulsh@mail.ahnu.edu.cn
}

Received 22 September 2016; Accepted 21 November 2016; Published 9 January 2017

Academic Editor: Yoshihiro Sawano

Copyright (C) 2017 Rulong Xie et al. This is an open access article distributed under the Creative Commons Attribution License, which permits unrestricted use, distribution, and reproduction in any medium, provided the original work is properly cited.

Let $(X, d, \mu)$ be a nonhomogeneous metric measure space. In this paper, the boundedness for commutators generated by bilinear $\theta$-type Calderón-Zygmund operators and $\operatorname{RBMO}(\mu)$ functions on $(X, d, \mu)$ is obtained.

\section{Introduction}

As we know, Hytönen [1] introduced nonhomogeneous metric measure spaces, which include both spaces of homogeneous type and nondoubling measure spaces as special cases. From then on, many results on nonhomogeneous metric measure spaces are obtained by many researchers. Hytönen et al. [2] and Bui and Duong [3] introduced independently the atomic Hardy space $H^{1}(\mu)$ and proved that the dual space of $H^{1}(\mu)$ is $\operatorname{RBMO}(\mu)$. The authors in [3] also proved that Calderón-Zygmund operator and commutators are bounded in $L^{p}(\mu)$ for $1<p<\infty$. Recently, some equivalent characterizations were established by Liu et al. [4] for the boundedness of Calderón-Zygmund operators on $L^{p}(\mu)$ for $1<p<\infty$. Fu et al. [5, 6] established boundedness of multilinear commutators of Calderón-Zygmund operators and generalized fractional integrals on Orlicz spaces, respectively. For more results, one can refer to $[2,4,7-15]$ and the references therein.

$\theta$-type Calderón-Zygmund operator was firstly introduced by Yabuta [16] in 1985. Later, many researchers further studied the properties of this operator. We [17] obtained the boundedness of $\theta$-type Calderón-Zygmund operator and commutators on nondoubling measure spaces. Ri et al.
$[18,19]$ researched the boundedness of $\theta$-type CalderónZygmund operator on Hardy spaces with nondoubling measures and nonhomogeneous metric measure spaces, respectively. Zheng et al. [20,21] studied the bounded properties for bilinear $\theta$-type Calderón-Zygmund operator and maximal bilinear $\theta$-type Calderón-Zygmund operator on nonhomogeneous metric measure spaces, respectively.

In this paper, we prepare to study the boundedness for commutators generated by bilinear $\theta$-type CalderónZygmund operators and $\operatorname{RBMO}(\mu)$ functions on nonhomogeneous metric measure spaces. And we obtain that these commutators are bounded on Lebesgue spaces, provided that bilinear $\theta$-type Calderón-Zygmund operator is bounded from $L^{1}(\mu) \times L^{1}(\mu)$ to $L^{1 / 2, \infty}(\mu)$, where $L^{p}(\mu)$ and $L^{p, \infty}(\mu)$ denote the Lebesgue spaces and weak Lebesgue spaces, respectively. This result includes the corresponding results on both spaces of homogeneous type and nondoubling measure spaces. It is even new in the settings of spaces of homogeneous type and nondoubling measure spaces.

Throughout this paper, $L_{c}^{\infty}(\mu)$ denotes the set of all $L^{\infty}(\mu)$ functions with compact support. $C$ always denotes a positive constant independent of the main parameters involved, but it may vary from line to line. And $p^{\prime}$ is the conjugate index of $p$; namely, $1 / p+1 / p^{\prime}=1$. Now, let us recall some definitions and terminologies. 
Definition 1 (see [1]). A metric space $(X, d)$ is geometrically doubling if there exists some $N_{0} \in \mathbf{N}$ such that, for every ball $B(x, r) \subset X$, there exists a finite ball covering $\left\{B\left(x_{i}, r / 2\right)\right\}_{i}$ of $B(x, r)$ such that the cardinality of this covering is at most $N_{0}$.

Definition 2 (see [1]). A metric measure space $(X, d, \mu)$ is upper doubling if $\mu$ is a Borel measure on $X$ and there exists a function $\lambda: X \times(0,+\infty) \rightarrow(0,+\infty)$ and a constant $C_{\lambda}>0$ such that, for every $x \in X, r \mapsto(x, r)$ is nondecreasing, and for any $x \in X, r>0$,

$$
\mu(B(x, r)) \leq \lambda(x, r) \leq C_{\lambda} \lambda\left(x, \frac{r}{2}\right)
$$

Remark 3. (i) Spaces of homogeneous type are upper doubling space, if we take $\lambda(x, r)=\mu(B(x, r))$. Also, nondoubling measure space, which satisfies the following polynomial growth condition:

$$
\mu(B(x, r)) \leq C r^{n}
$$

for all $x \in \mathbb{R}^{d}$ and $r>0$, is also upper doubling measure space if we take $\lambda(x, r)=C r^{n}$.

(ii) The authors [11] showed that there exists another function $\widetilde{\lambda}$ such that, for any $x, y \in X, d(x, y) \leq r$,

$$
\tilde{\lambda}(x, r) \leq \widetilde{C} \tilde{\lambda}(y, r) .
$$

Thus, one assumes that $\lambda$ always satisfies (3) in this paper. As the singularity of commutators is stronger than that of bilinear operators, by [22], we suppose that there exists $0<$ $m<+\infty$, such that, for any $a, r>0, x \in X$,

$$
\lambda(x, a r) \geq a^{m} \lambda(x, r) .
$$

Let $\alpha, \beta \in(1,+\infty)$; a ball $B \subset X$ is $(\alpha, \beta)$-doubling if $\mu(\alpha B) \leq \beta \mu(B)$. As pointed in Lemma 2.3 of [3], there exist plenty of doubling balls with small radii and with large radii. In this paper, unless $\alpha$ and $\beta$ are specified otherwise, one means $(\alpha, \beta)$ doubling ball is $\left(6, \beta_{0}\right)$-doubling with a fixed number $\beta_{0}>\max \left\{C_{\lambda}^{3 \log _{2} 6}, 6^{n}\right\}$, where $n=\log _{2} N_{0}$ is the geometric dimension of the space.

Definition 4 (see [3]). For two balls $B \subset Q$, let $N_{B, Q}$ be the smallest integer such that $6^{N_{B, Q}} r_{B} \geq r_{Q}$; we denote

$$
K_{B, Q}=1+\sum_{k=1}^{N_{B, Q}} \frac{\mu\left(6^{k} B\right)}{\lambda\left(c_{B}, 6^{k} r_{B}\right)} \text {. }
$$

Let $\theta$ be a nonnegative nondecreasing function on $(0,+\infty)$ satisfying

$$
\int_{0}^{1} \frac{\theta(t)}{t}|\log t| d t<\infty
$$

Definition 5. A kernel $K(\cdot, \cdot, \cdot) \in L_{\text {loc }}^{1}\left(X^{3} \backslash\left\{\left(x, y_{1}, y_{2}\right): x=\right.\right.$ $\left.\left.y_{1}=y_{2}\right\}\right)$ is called the bilinear $\theta$-type Calderón-Zygmund kernel if it satisfies the following: (i)

$$
\left|K\left(x, y_{1}, y_{2}\right)\right| \leq C\left[\sum_{j=1}^{2} \lambda\left(x, d\left(x, y_{j}\right)\right)\right]^{-2}
$$

for all $\left(x, y_{1}, y_{2}\right) \in X^{3}$ with $x \neq y_{j}$ for $j \in\{1,2\}$.

(ii)

$$
\begin{aligned}
& \left|K\left(x, y_{1}, y_{2}\right)-K\left(x^{\prime}, y_{1}, y_{2}\right)\right| \\
& \quad \leq C \theta\left(\frac{d\left(x, x^{\prime}\right)}{\sum_{j=1}^{2} d\left(x, y_{j}\right)}\right)\left[\sum_{j=1}^{2} \lambda\left(x, d\left(x, y_{j}\right)\right)\right]^{-2}
\end{aligned}
$$

provided that $C d\left(x, x^{\prime}\right) \leq \max _{1 \leq j \leq 2} d\left(x, y_{j}\right)$.

A bilinear operator $T_{\theta}$ is called bilinear $\theta$-type CalderónZygmund operator with the above kernel $K$ if for $f_{1}, f_{2} \in L_{c}^{\infty}$ and $x \notin \bigcap_{j=1}^{2} \operatorname{supp} f_{j}$,

$$
\begin{aligned}
T_{\theta} & \left(f_{1}, f_{2}\right)(x) \\
& =\int_{X^{2}} K\left(x, y_{1}, y_{2}\right) f_{1}\left(y_{1}\right) f_{2}\left(y_{2}\right) d \mu\left(y_{1}\right) d \mu\left(y_{2}\right) .
\end{aligned}
$$

Remark 6. As $\max _{1 \leq j \leq 2} d\left(x, y_{j}\right) \leq \sum_{j=1}^{2} d\left(x, y_{j}\right) \leq$ $2 \max _{1 \leq j \leq 2} d\left(x, y_{j}\right)$, (ii) in Definition 5 is equivalent to (ii') in the following statement:

$\left(\mathrm{ii}^{\prime}\right)$

$$
\begin{aligned}
& \left|K\left(x, y_{1}, y_{2}\right)-K\left(x^{\prime}, y_{1}, y_{2}\right)\right| \\
& \leq C \theta\left(\frac{d\left(x, x^{\prime}\right)}{\max _{1 \leq j \leq 2} d\left(x, y_{j}\right)}\right)\left[\sum_{j=1}^{2} \lambda\left(x, d\left(x, y_{j}\right)\right)\right]^{-2} \\
& \text { provided that } C d\left(x, x^{\prime}\right) \leq \max _{1 \leq j \leq 2} d\left(x, y_{j}\right) .
\end{aligned}
$$

Remark 7. (i) In $[20,21]$, the term $\left[\sum_{j=1}^{2} \lambda\left(x, d\left(x, y_{j}\right)\right)\right]^{-2}$ in (7) and (8) of this paper is substituted by $\min _{j \in\{1,2\}}[\lambda(x$, $\left.\left.d\left(x, y_{j}\right)\right)\right]^{-2}$. In fact, as

$$
\begin{aligned}
\min _{j \in\{1,2\}}\left[\lambda\left(x, d\left(x, y_{j}\right)\right)\right]^{-2} \\
=\left[\lambda\left(x, \max _{j \in\{1,2\}} d\left(x, y_{j}\right)\right)\right]^{-2}
\end{aligned}
$$

and $\lambda\left(x, \max _{j \in\{1,2\}} d\left(x, y_{j}\right)\right) \leq \sum_{j=1}^{2} \lambda\left(x, d\left(x, y_{j}\right)\right) \leq 2 \lambda(x$, $\left.\max _{j \in\{1,2\}} d\left(x, y_{j}\right)\right)$, Definition 5 in this paper is equivalent to Definition 1.4 in [20] or Definition 1.3 in [21]. Therefore, we can directly quote the result of Theorem 1.5 in [20] as Lemma 17 below in this paper.

(ii) Because we assume that $T_{\theta}$ is bounded from $L^{1}(\mu) \times$ $L^{1}(\mu)$ to $L^{1 / 2, \infty}(\mu)$, it is enough to assume that $K$ satisfies the regularity condition on the first variable, that is, (8) in this paper for getting the result of Theorem 10 below. For more details, one can refer to Remark 1.1 in [9]. 
Definition 8 . The commutator generated by bilinear $\theta$-type Calderón-Zygmund operator $T_{\theta}$ and $b_{1}, b_{2} \in \operatorname{RBMO}(\mu)$ is defined by

$$
\begin{aligned}
& {\left[b_{1}, b_{2}, T_{\theta}\right]\left(f_{1}, f_{2}\right)(x)} \\
& =b_{1}(x) b_{2}(x) T_{\theta}\left(f_{1}, f_{2}\right)(x) \\
& \quad-b_{1}(x) T_{\theta}\left(f_{1}, b_{2} f_{2}\right)(x) \\
& \quad-b_{2}(x) T_{\theta}\left(b_{1} f_{1}, f_{2}\right)(x)+T_{\theta}\left(b_{1} f_{1}, b_{2} f_{2}\right)(x) .
\end{aligned}
$$

Also, $\left[b_{1}, T_{\theta}\right]$ and $\left[b_{2}, T_{\theta}\right]$ are defined as follows, respectively:

$$
\begin{array}{r}
{\left[b_{1}, T_{\theta}\right]\left(f_{1}, f_{2}\right)(x)=b_{1}(x) T_{\theta}\left(f_{1}, f_{2}\right)(x)} \\
-T_{\theta}\left(b_{1} f_{1}, f_{2}\right)(x), \\
{\left[b_{2}, T_{\theta}\right]\left(f_{1}, f_{2}\right)(x)=} \\
b_{2}(x) T_{\theta}\left(f_{1}, f_{2}\right)(x) \\
-T_{\theta}\left(f_{1}, b_{2} f_{2}\right)(x) .
\end{array}
$$

Definition 9 (see [2]). Let $\rho>1$ be some fixed constant. A function $b \in L_{\text {loc }}^{1}(\mu)$ is said to belong to $\operatorname{RBMO}(\mu)$ if there exists a constant $C>0$ such that, for any ball $B$,

$$
\frac{1}{\mu(\rho B)} \int_{B}\left|b(x)-m_{\widetilde{B}}(b)\right| d \mu(x) \leq C,
$$

and for any two doubling balls $B \subset Q$,

$$
\left|m_{B}(b)-m_{\mathrm{Q}}(b)\right| \leq C K_{B, Q},
$$

where $\widetilde{B}$ is the smallest $(\alpha, \beta)$-doubling ball of the form $6^{k} B$ with $k \in \mathbf{N} \cup\{0\}$, and $m_{\widetilde{B}}(b)$ is the mean value of $b$ on $\widetilde{B}$ : namely,

$$
m_{\widetilde{B}}(b)=\frac{1}{\mu(\widetilde{B})} \int_{\widetilde{B}} b(x) d \mu(x) .
$$

The minimal constant $C$ in (15) and (16) is the $\operatorname{RBMO}(\mu)$ norm of $b$, which is denoted by $\|b\|_{*}$.

Theorem 10. Let $1<p_{1}, p_{2}<+\infty, 1 / q=1 / p_{1}+1 / p_{2}, b_{1}, b_{2} \in$ $R B M O(\mu)$. Assume that $f_{1} \in L^{p_{1}}(\mu), f_{2} \in L^{p_{2}}(\mu)$ with $\int_{X} T_{\theta}\left(f_{1}, f_{2}\right)(x) d \mu(x)=0, \int_{X}\left[b_{1}, T_{\theta}\right]\left(f_{1}, f_{2}\right)(x) d \mu(x)=0$, $\int_{X}\left[b_{2}, T_{\theta}\right]\left(f_{1}, f_{2}\right)(x) d \mu(x)=0, \int_{X}\left[b_{1}, b_{2}, T_{\theta}\right]\left(f_{1}, f_{2}\right)(x) d \mu(x)$ $=0$ if $\|\mu\|<\infty$. If $T_{\theta}$ is bounded from $L^{1}(\mu) \times L^{1}(\mu)$ to $L^{1 / 2, \infty}(\mu)$, then there exists a constant $C>0$ such that

$$
\left\|\left[b_{1}, b_{2}, T_{\theta}\right]\left(f_{1}, f_{2}\right)\right\|_{L^{q}(\mu)} \leq C\left\|f_{1}\right\|_{L^{p_{1}}(\mu)}\left\|f_{2}\right\|_{L^{p_{2}}(\mu)} .
$$

Remark 11. The result of Theorem 10 is still valid for commutators of multilinear $\theta$-type Calderón-Zygmund operators with $\operatorname{RBMO}(\mu)$ functions.

\section{Preliminaries}

For any $f \in L_{\text {loc }}^{1}(\mu)$, the noncentered doubling maximal operator is defined by

$$
N f(x)=\sup _{\substack{B \ni x, B \text { doubling }}} \frac{1}{\mu(B)} \int_{B}|f(y)| d \mu(y),
$$

and the sharp maximal operator $M^{\sharp}$ is denoted by

$$
\begin{aligned}
M^{\sharp} f(x)= & \sup _{B \ni x} \frac{1}{\mu(6 B)} \int_{B}\left|f(y)-m_{\widetilde{B}}(f)\right| d \mu(y) \\
& +\sup _{(B, Q) \in \Delta_{x}} \frac{\left|m_{B}(f)-m_{Q}(f)\right|}{K_{B, Q}},
\end{aligned}
$$

where $\Delta_{x}:=\{(B, Q): x \in B \subset Q$ and $B, Q$ are doubling balls $\}$.

For any $0<\eta<1$, denote

$$
\begin{aligned}
& N_{\eta} f(x)=\left\{N\left(|f|^{\eta}\right)(x)\right\}^{1 / \eta}, \\
& M_{\eta}^{\sharp} f(x)=\left\{M^{\sharp}\left(|f|^{\eta}\right)(x)\right\}^{1 / \eta} .
\end{aligned}
$$

Let $\rho>1, p \in(1, \infty)$, and $s \in(1, p)$; the noncentered maximal operator $M_{s,(\rho)} f$ is defined by

$$
M_{s,(\rho)} f(x)=\sup _{B \ni x}\left\{\frac{1}{\mu(\rho B)} \int_{B}|f(y)|^{r} d \mu(y)\right\}^{1 / s} .
$$

When $s=1$, we simply write $M_{1,(\rho)} f(x)$ as $M_{(\rho)} f$.

Lemma 12 (see $[3,11])$. (i) For any $f \in L_{\mathrm{loc}}^{1}(\mu)$ and $\mu$-a.e. $x \in X$,

$$
|f(x)| \leq N_{\eta} f(x) .
$$

(ii) If $\rho \geq 5$, then the operator $M_{(\rho)} f$ is bounded on $L^{p}(\mu)$ for $p>1$ and $M_{s,(\rho)}$ is bounded on $L^{p}(\mu)$ for $p>s>1$.

Lemma 13 (see $[3,5]$ ). Assume that $f \in L_{\text {loc }}^{1}(\mu)$ with $\int_{X} f(x) d \mu(x)=0$ if $\|\mu\|<\infty$. For $1<p<\infty$ and $0<\eta<1$, if $\inf \left(1, N_{\eta} f\right) \in L^{p}(\mu)$, then there exists a constant $C>0$ such that

$$
\left\|N_{\eta}(f)\right\|_{L^{p}(\mu)} \leq C\left\|M_{\eta}^{\sharp}(f)\right\|_{L^{p}(\mu)} .
$$

Lemma 14 (see $[5,23])$. Suppose that $1 \leq p<\infty$ and $1<\rho<$ $\infty$. Then $b \in R B M O(\mu)$ if and only if for any ball $B \subset X$,

$$
\left\{\frac{1}{\mu(\rho B)} \int_{B}\left|b_{B}-m_{\widetilde{B}}(b)\right|^{p} d \mu(x)\right\}^{1 / p} \leq C\|b\|_{*},
$$

and for any two doubling balls $B \subset Q$,

$$
\left|m_{B}(b)-m_{Q}(b)\right| \leq C K_{B, Q}\|b\|_{*} .
$$

Lemma 15 (see [5]). For any $k \in \mathbb{N}^{+}$,

$$
\left|m_{6^{k}(6 / 5) B}(b)-m_{\widetilde{B}}(b)\right| \leq C k\|b\|_{*} .
$$

Lemma 16 (Kolmogorov's theorem). Let $(X, \mu)$ be a probability measure space and let $0<p<q<\infty$; then there exists a constant $C>0$, such that $\|f\|_{L^{p}(\mu)} \leq C\|f\|_{L^{q, \infty}(\mu)}$ for any measurable function $f$.

Lemma 17 (see [20]). Let $1<p_{1}, p_{2}<+\infty, 1 / q=1 / p_{1}+$ $1 / p_{2}, f_{1} \in L^{p_{1}}(\mu)$, and $f_{2} \in L^{p_{2}}(\mu)$. If $T_{\theta}$ is bounded from $L^{1}(\mu) \times L^{1}(\mu)$ to $L^{1 / 2, \infty}(\mu)$, then there exists a constant $C>0$ such that

$$
\left\|T_{\theta}\left(f_{1}, f_{2}\right)\right\|_{L^{q}(\mu)} \leq C\left\|f_{1}\right\|_{L^{p_{1}}(\mu)}\left\|f_{2}\right\|_{L^{p_{2}}(\mu)} .
$$




\section{Proof of Main Result}

Lemma 18. Suppose that $0<\eta<1 / 2,1<p_{1}, p_{2}, q<\infty, 1<$ $s<q$ and $b_{1}, b_{2} \in R B M O(\mu)$. If $T_{\theta}$ is bounded from $L^{1}(\mu) \times$ $L^{1}(\mu)$ to $L^{1 / 2, \infty}(\mu)$, then there exists a constant $C>0$ such that, for any $x \in X, f_{1} \in L^{p_{1}}(\mu)$, and $f_{2} \in L^{p_{2}}(\mu)$,

$$
\begin{aligned}
& M_{\eta}^{\sharp} {\left[b_{1}, b_{2}, T_{\theta}\right]\left(f_{1}, f_{2}\right)(x) } \\
& \leq C\left\|b_{1}\right\|_{*}\left\|b_{2}\right\|_{*} M_{s,(6)}\left(T_{\theta}\left(f_{1}, f_{2}\right)\right)(x) \\
&+C\left\|b_{1}\right\|_{*} M_{s,(6)}\left(\left[b_{2}, T_{\theta}\right]\left(f_{1}, f_{2}\right)\right)(x) \\
&+C\left\|b_{2}\right\|_{*} M_{s,(6)}\left(\left[b_{1}, T_{\theta}\right]\left(f_{1}, f_{2}\right)\right)(x) \\
&+C\left\|b_{1}\right\|_{*}\left\|b_{2}\right\|_{*} M_{p_{1},(5)} f_{1}(x) M_{p_{2},(5)} f_{2}(x), \\
& M_{\eta}^{\sharp}\left[b_{1}, T_{\theta}\right]\left(f_{1}, f_{2}\right)(x) \\
& \leq C\left\|b_{1}\right\|_{*} M_{s,(6)}\left(T_{\theta}\left(f_{1}, f_{2}\right)\right)(x) \\
&+C\left\|b_{1}\right\|_{*} M_{p_{1},(5)} f_{1}(x) M_{p_{2},(5)} f_{2}(x), \\
& M_{\eta}^{\sharp} {\left[b_{2}, T_{\theta}\right]\left(f_{1}, f_{2}\right)(x) } \\
& \leq C\left\|b_{2}\right\|_{*} M_{s,(6)}\left(T_{\theta}\left(f_{1}, f_{2}\right)\right)(x) \\
&+C\left\|b_{2}\right\|_{*} M_{p_{1},(5)} f_{1}(x) M_{p_{2},(5)} f_{2}(x) .
\end{aligned}
$$

Proof. Because $L_{c}^{\infty}(\mu)$ is dense in $L^{p}(\mu)$ for $1<p<\infty$, we only consider the situation of $f_{1}, f_{2} \in L_{c}^{\infty}(\mu)$. Also, by Lemma 3.11 in [5], we can assume that $b_{1}, b_{2} \in L^{\infty}(\mu)$. As it has the similar method to estimate (29), (30), and (31), here we only estimate (29) for complicity.

To obtain (29), with the similar way to prove Theorem 9.1 in [24], it suffices to show that

$$
\begin{aligned}
& \left(\frac{1}{\mu(6 B)}\right. \\
& \left.\cdot \int_{B} \|\left.\left[b_{1}, b_{2}, T_{\theta}\right]\left(f_{1}, f_{2}\right)(z)\right|^{\eta}-\left|h_{B}\right|^{\eta} \mid d \mu(z)\right)^{1 / \eta} \\
& \quad \leq C\left\|b_{1}\right\|_{*}\left\|b_{2}\right\|_{*} M_{s,(6)}\left(T_{\theta}\left(f_{1}, f_{2}\right)\right)(x)+C\left\|b_{1}\right\|_{*} \\
& \cdot M_{s,(6)}\left(\left[b_{2}, T_{\theta}\right]\left(f_{1}, f_{2}\right)\right)(x)+C\left\|b_{2}\right\|_{*} \\
& \cdot M_{s,(6)}\left(\left[b_{1}, T_{\theta}\right]\left(f_{1}, f_{2}\right)\right)(x)+C\left\|b_{1}\right\|_{*}\left\|b_{2}\right\|_{*} \\
& \cdot M_{p_{1},(5)} f_{1}(x) M_{p_{2},(5)} f_{2}(x)
\end{aligned}
$$

holds for any $x \in B$, and

$$
\begin{aligned}
& \left|h_{B}-h_{\mathrm{Q}}\right| \\
& \quad \leq C K_{B, \mathrm{Q}}^{2}\left[\left\|b_{1}\right\|_{*}\left\|b_{2}\right\|_{*} M_{s,(6)}\left(T_{\theta}\left(f_{1}, f_{2}\right)\right)(x)\right. \\
& \quad+\left\|b_{1}\right\|_{*} M_{s,(6)}\left(\left[b_{2}, T_{\theta}\right]\left(f_{1}, f_{2}\right)\right)(x) \\
& \quad+\left\|b_{2}\right\|_{*} M_{s,(6)}\left(\left[b_{1}, T_{\theta}\right]\left(f_{1}, f_{2}\right)\right)(x) \\
& \left.\quad+\left\|b_{1}\right\|_{*}\left\|b_{2}\right\|_{*} M_{p_{1},(5)} f_{1}(x) M_{p_{2},(5)} f_{2}(x)\right]
\end{aligned}
$$

$$
\begin{aligned}
& \left(\left.\frac{1}{\mu(6 B)} \int_{B}||\left[b_{1}, b_{2}, T_{\theta}\right]\left(f_{1}, f_{2}\right)(z)\right|^{\eta}-\left|h_{B}\right|^{\eta} \mid d \mu(z)\right)^{1 / \eta} \\
& \leq C\left(\frac{1}{\mu(6 B)} \int_{B} \mid\left(b_{1}(z)-m_{\widetilde{B}}\left(b_{1}\right)\right)\left(b_{2}(z)-m_{\tilde{B}}\left(b_{2}\right)\right)\right. \\
& \left.\left.\cdot T_{\theta}\left(f_{1}, f_{2}\right)(z)\right|^{\eta} d \mu(z)\right)^{1 / \eta}+C\left(\frac{1}{\mu(6 B)}\right. \\
& \cdot \int_{B} \mid\left(b_{1}(z)-m_{\widetilde{B}}\left(b_{1}\right)\right) T_{\theta}\left(f_{1},\left(b_{2}-b_{2}(z)\right) f_{2}\right) \\
& \left.\left.\cdot(z)\right|^{\eta} d \mu(z)\right)^{1 / \eta}+C\left(\frac{1}{\mu(6 B)}\right. \\
& \cdot \int_{B} \mid\left(b_{2}(z)-m_{\widetilde{B}}\left(b_{2}\right)\right) T_{\theta}\left(\left(b_{1}-b_{1}(z)\right) f_{1}, f_{2}\right) \\
& \left.\left.\cdot(z)\right|^{\eta} d \mu(z)\right)^{1 / \eta}+C\left(\frac{1}{\mu(6 B)}\right. \\
& \cdot \int_{B} \mid T_{\theta}\left(\left(b_{1}-m_{\tilde{B}}\left(b_{1}\right)\right) f_{1},\left(b_{2}-m_{\widetilde{B}}\left(b_{2}\right)\right) f_{2}\right)(z) \\
& \left.-\left.h_{B}\right|^{\eta} d \mu(z)\right)^{1 / \eta}=: I+I I+I I I+I V .
\end{aligned}
$$


Journal of Function Spaces

5

For $I$. Let $s_{1}, s_{2}>1$ such that $1 / s_{1}+1 / s_{2}+1 / s=1 / \eta$. By Hölder's inequality and Lemma 14,

$$
\begin{aligned}
I \leq & C\left(\frac{1}{\mu(6 B)} \int_{B}\left|b_{1}(z)-m_{\widetilde{B}}\left(b_{1}\right)\right|^{s_{1}} d \mu(z)\right)^{1 / s_{1}} \\
& \times\left(\frac{1}{\mu(6 B)} \int_{B}\left|b_{2}(z)-m_{\widetilde{B}}\left(b_{2}\right)\right|^{s_{2}} d \mu(z)\right)^{1 / s_{2}} \\
& \times\left(\frac{1}{\mu(6 B)} \int_{B}\left|T_{\theta}\left(f_{1}, f_{2}\right)\right|^{s} d \mu(z)\right)^{1 / s} \\
\leq & C\left\|b_{1}\right\|_{*}\left\|b_{2}\right\|_{*} M_{s,(6)}\left(T_{\theta}\left(f_{1}, f_{2}\right)\right)(x) .
\end{aligned}
$$

Let us estimate $I I$, let $t>1$ such that $1 / t+1 / s=1 / \eta$, and then

$$
\begin{aligned}
I I \leq & C\left(\frac{1}{\mu(6 B)} \int_{B}\left|b_{1}(z)-m_{\widetilde{B}}\left(b_{1}\right)\right|^{t} d \mu(z)\right)^{1 / t} \\
& \times\left(\frac{1}{\mu(6 B)} \int_{B}\left|\left[b_{2}, T_{\theta}\right]\left(f_{1}, f_{2}\right)\right|^{s} d \mu(z)\right)^{1 / s} \\
\leq & C\left\|b_{1}\right\|_{*} M_{s,(6)}\left(\left[b_{2}, T_{\theta}\right]\left(f_{1}, f_{2}\right)\right)(x) .
\end{aligned}
$$

Similar to estimate $I I$,

$$
I I I \leq C\left\|b_{2}\right\|_{*} M_{s,(6)}\left(\left[b_{1}, T_{\theta}\right]\left(f_{1}, f_{2}\right)\right)(x) .
$$

Let us turn to estimate $I V$. Let $f_{j}^{1}=f_{j} \chi_{(6 / 5) B}, f_{j}^{2}=f_{j}-f_{j}^{1}$ for $j \in\{1,2\}$; then

$$
\begin{aligned}
I V & \leq C\left(\frac{1}{\mu(6 B)}\right. \\
\cdot & \int_{B} \mid T_{\theta}\left(\left(b_{1}-m_{\widetilde{B}}\left(b_{1}\right)\right) f_{1}^{1}(z),\left(b_{2}-m_{\widetilde{B}}\left(b_{2}\right)\right) f_{2}^{1}\right) \\
\cdot & \left.\left.(z)\right|^{\eta} d \mu(z)\right)^{1 / \eta}+C\left(\frac{1}{\mu(6 B)}\right. \\
& \cdot \int_{B} \mid T_{\theta}\left(\left(b_{1}-m_{\widetilde{B}}\left(b_{1}\right)\right) f_{1}^{1}(z),\left(b_{2}-m_{\widetilde{B}}\left(b_{2}\right)\right) f_{2}^{2}\right) \\
& \left.\left.\cdot(z)\right|^{\eta} d \mu(z)\right)^{1 / \eta}+C\left(\frac{1}{\mu(6 B)}\right. \\
& \cdot \int_{B} \mid T_{\theta}\left(\left(b_{1}-m_{\widetilde{B}}\left(b_{1}\right)\right) f_{1}^{2}(z),\left(b_{2}-m_{\widetilde{B}}\left(b_{2}\right)\right) f_{2}^{1}\right) \\
& \left.\left.\cdot(z)\right|^{\eta} d \mu(z)\right)^{1 / \eta}+C\left(\frac{1}{\mu(6 B)}\right. \\
& \cdot \int_{B} \mid T_{\theta}\left(\left(b_{1}-m_{\widetilde{B}}\left(b_{1}\right)\right) f_{1}^{2}(z),\left(b_{2}-m_{\widetilde{B}}\left(b_{2}\right)\right) f_{2}^{2}\right)(z) \\
& \left.-\left.h_{B}\right|^{\eta} d \mu(z)\right)^{1 / \eta}=: I V_{1}+I V_{2}+I V_{3}+I V_{4} .
\end{aligned}
$$




$$
\begin{aligned}
& \left.\times \int_{6^{k+1}(6 / 5) B}\left|b_{2}\left(y_{2}\right)-m_{6^{k}(6 / 5) B}\left(b_{2}\right)\right|^{p_{2}^{\prime}} d \mu\left(y_{2}\right)\right)^{1 / p_{2}^{\prime}} \\
& \times\left(\frac{1}{\mu\left(5 \times 6^{k}(6 / 5) B\right)}\right. \\
& \left.\cdot \int_{6^{k}(6 / 5) B}\left|f_{2}\left(y_{2}\right)\right|^{p_{2}} d \mu\left(y_{2}\right)\right)^{1 / p_{2}}+C k\left\|b_{2}\right\|_{*}
\end{aligned}
$$$$
\left.\frac{1}{\mu\left(5 \times 6^{k}(6 / 5) B\right)} \int_{\sigma^{k}(6 / 5) B}\left|f_{2}\left(y_{2}\right)\right| d \mu\left(y_{2}\right)\right]
$$$$
\leq C\left\|b_{1}\right\|_{*}\left\|b_{2}\right\|_{*} M_{p_{1},(5)} f_{1}(x) M_{p_{2},(5)} f_{2}(x) .
$$

Similarly, we obtain

$$
I V_{3} \leq C\left\|b_{1}\right\|_{*}\left\|b_{2}\right\|_{*} M_{p_{1},(5)} f_{1}(x) M_{p_{2},(5)} f_{2}(x) .
$$

For $I V_{4}$, by (ii) of Definition 5 and some properties of $\lambda$,

$$
\begin{aligned}
& \left|T_{\theta}\left(\left(b_{1}-m_{\widetilde{B}}\left(b_{1}\right)\right) f_{1}^{2},\left(b_{2}-m_{\widetilde{B}}\left(b_{2}\right)\right) f_{2}^{2}\right)(z)-T_{\theta}\left(\left(b_{1}-m_{\widetilde{B}}\left(b_{1}\right)\right) f_{1}^{2},\left(b_{2}-m_{\widetilde{B}}\left(b_{2}\right)\right) f_{2}^{2}\right)\left(z_{0}\right)\right| \\
& \quad \leq C \int_{X \backslash(6 / 5) B} \int_{X \backslash(6 / 5) B}\left|K\left(z, y_{1}, y_{2}\right)-K\left(z_{0}, y_{1}, y_{2}\right)\right| \times\left|\prod_{i=1}^{2}\left(b_{i}\left(y_{i}\right)-m_{\widetilde{B}}\left(b_{i}\right)\right) f_{i}\left(y_{i}\right)\right| d \mu\left(y_{1}\right) d \mu\left(y_{2}\right) \\
& \quad \leq C \sum_{j=1}^{\infty} \sum_{k=j}^{\infty} \int_{\sigma^{k}(6 / 5) B \backslash 6^{k-1}(6 / 5) B} \theta\left(\frac{d\left(z, z_{0}\right)}{d\left(z, y_{1}\right)}\right)\left|\left(b_{1}\left(y_{1}\right)-m_{\widetilde{B}}\left(b_{1}\right)\right) f_{1}\left(y_{1}\right)\right| \\
& \quad \times \int_{6^{j}(6 / 5) B \backslash \sigma^{j-1}(6 / 5) B} \frac{\left|\left(b_{2}\left(y_{2}\right)-m_{\widetilde{B}}\left(b_{2}\right)\right) f_{2}\left(y_{2}\right)\right|}{\left[\lambda\left(z, d\left(z, y_{2}\right)\right)\right]^{2}} d \mu\left(y_{2}\right) d \mu\left(y_{1}\right) \\
& \quad+C \sum_{j=1}^{\infty} \sum_{k=1}^{j-1} \int_{\sigma^{j}(6 / 5) B \backslash 6^{j-1}(6 / 5) B} \theta\left(\frac{d\left(z, z_{0}\right)}{d\left(z, y_{2}\right)}\right)\left|\left(b_{2}\left(y_{2}\right)-m_{\widetilde{B}}\left(b_{2}\right)\right) f_{2}\left(y_{2}\right)\right| \\
& \quad \times \int_{6^{k}(6 / 5) B \backslash 6^{k-1}(6 / 5) B} \frac{\left|\left(b_{1}\left(y_{1}\right)-m_{\widetilde{B}}\left(b_{1}\right)\right) f_{1}\left(y_{1}\right)\right|}{\left[\lambda\left(z, d\left(z, y_{1}\right)\right)\right]^{2}} d \mu\left(y_{1}\right) d \mu\left(y_{2}\right)=: I V_{41}+I V_{42} .
\end{aligned}
$$

Let us estimate $I V_{41}$. With the help of the fact that

by Lemmas 14 and 15 and Hölder's inequality, we have

$$
\begin{aligned}
\int_{0}^{1} \frac{\theta(t)}{t}|\log t| d t & \geq \sum_{k=1}^{\infty} \int_{6^{-k}}^{6^{1-k}} \frac{\theta\left(6^{-k}\right)}{6^{1-k}}\left|\log 6^{-k}\right| d t \\
& \geq C \sum_{k=1}^{\infty} k \theta\left(6^{-k}\right)
\end{aligned}
$$

$$
\begin{aligned}
& I V_{41} \leq C \sum_{k=1}^{\infty} \frac{1}{\left[\lambda\left(z, 6^{k}(6 / 5) r_{B}\right)\right]^{2}} \int_{6^{k}(6 / 5) B} \theta\left(6^{-k}\right)\left|\left(b_{1}\left(y_{1}\right)-m_{\widetilde{B}}\left(b_{1}\right)\right) f_{1}\left(y_{1}\right)\right| d \mu\left(y_{1}\right) \\
& \quad \times \sum_{j=1}^{k} \int_{\sigma^{j}(6 / 5) B \mid 6^{j-1}(6 / 5) B}\left|\left(b_{2}\left(y_{2}\right)-m_{\widetilde{B}}\left(b_{2}\right)\right) f_{2}\left(y_{2}\right)\right| d \mu\left(y_{2}\right) \leq C \sum_{k=1}^{\infty} \theta\left(6^{-k}\right) \frac{1}{\left[\lambda\left(z, 6^{k}(6 / 5) r_{B}\right)\right]^{2}} \\
& \quad \cdot \int_{6^{k}(6 / 5) B}\left|\left(b_{1}\left(y_{1}\right)-m_{\widetilde{B}}\left(b_{1}\right)\right) f_{1}\left(y_{1}\right)\right| d \mu\left(y_{1}\right) \times \int_{6^{k}(6 / 5) B}\left|\left(b_{2}\left(y_{2}\right)-m_{\widetilde{B}}\left(b_{2}\right)\right) f_{2}\left(y_{2}\right)\right| d \mu\left(y_{2}\right) \leq C \sum_{k=1}^{\infty} \theta\left(6^{-k}\right) \\
& \quad \cdot \prod_{i=1}^{2}\left(\frac{1}{\mu\left(5 \times 6^{k}(6 / 5) B\right)} \int_{6^{k}(6 / 5) B}\left|b_{i}\left(y_{i}\right)-m_{\widetilde{B}}\left(b_{i}\right)\right|^{p_{i}^{\prime}} d \mu\left(y_{i}\right)\right)^{1 / p_{i}^{\prime}} \times\left(\frac{1}{\mu\left(5 \times 6^{k}(6 / 5) B\right)} \int_{6^{k}(6 / 5) B}\left|f_{i}\left(y_{i}\right)\right|^{p_{i}}\right)^{1 / p_{i}}
\end{aligned}
$$




$$
\begin{aligned}
& \leq C \sum_{k=1}^{\infty} \theta\left(6^{-k}\right) \prod_{i=1}^{2} M_{p_{i},(6)} f_{i}(x) \\
& \cdot\left(\frac{1}{\mu\left(5 \times 6^{k}(6 / 5) B\right)} \int_{\sigma^{k}(6 / 5) B}\left|b_{i}\left(y_{i}\right)-m_{6^{k}(6 / 5) B}\left(b_{i}\right)+m_{\sigma^{k}(6 / 5) B}\left(b_{i}\right)-m_{\widetilde{B}}\left(b_{i}\right)\right|^{p_{i}^{\prime}} d \mu\left(y_{i}\right)\right)^{1 / p_{i}^{\prime}} \leq C \sum_{k=1}^{\infty} \theta\left(6^{-k}\right) k \\
& \cdot \prod_{i=1}^{2}\left\|b_{i}\right\|_{*} M_{p_{i},(5)} f_{i}(x) \leq C \int_{0}^{1} \frac{\theta(t)}{t}|\log t| d t \prod_{i=1}^{2}\left\|b_{i}\right\|_{*} M_{p_{i},(5)} f_{i}(x) \leq C\left\|b_{1}\right\|_{*}\left\|b_{2}\right\|_{*} M_{p_{1},(5)} f_{1}(x) M_{p_{2},(5)} f_{2}(x) .
\end{aligned}
$$

With the same method to estimate $I V_{41}$,

$$
I V_{42} \leq C\left\|b_{1}\right\|_{*}\left\|b_{2}\right\|_{*} M_{p_{1},(5)} f_{1}(x) M_{p_{2},(5)} f_{2}(x) .
$$

Thus, taking the mean over $z_{0} \in B$, we have

$$
I V_{4} \leq C\left\|b_{1}\right\|_{*}\left\|b_{2}\right\|_{*} M_{p_{1},(5)} f_{1}(x) M_{p_{2},(5)} f_{2}(x) .
$$

So (32) can be obtained.

Next we prove (33). Denote $N=N_{B, Q}+1$; then

$$
\begin{aligned}
& || m_{B} T_{\theta}\left(\left(b_{1}-m_{\tilde{B}}\left(b_{1}\right)\right) f_{1}^{2},\left(b_{2}-m_{\tilde{B}}\left(b_{2}\right)\right) f_{2}^{2}\right) \mid \\
& \text { - }\left|m_{\mathrm{Q}} T_{\theta}\left(\left(b_{1}-m_{\mathrm{Q}}\left(b_{1}\right)\right) f_{1}^{2},\left(b_{2}-m_{\mathrm{Q}}\left(b_{2}\right)\right) f_{2}^{2}\right)\right| \mid \\
& \leq \mid m_{B} T_{\theta}\left(\left(b_{1}-m_{\widetilde{B}}\left(b_{1}\right)\right) f_{1} \chi_{X \mid 6^{N} B},\left(b_{2}-m_{\widetilde{B}}\left(b_{2}\right)\right)\right. \\
& \text { - } \left.f_{2} \chi_{X \mid \sigma^{N} B}\right)-m_{\mathrm{Q}} T_{\theta}\left(\left(b_{1}-m_{\widetilde{B}}\left(b_{1}\right)\right)\right. \\
& \text { - } \left.f_{1} \chi_{X \backslash 6^{N} B},\left(b_{2}-m_{\widetilde{B}}\left(b_{2}\right)\right) f_{2} \chi_{X \backslash 6^{N} B}\right) \mid \\
& +\mid m_{\mathrm{Q}} T_{\theta}\left(\left(b_{1}-m_{\mathrm{Q}}\left(b_{1}\right)\right) f_{1} \chi_{X \backslash 6^{N} B},\left(b_{2}-m_{\mathrm{Q}}\left(b_{2}\right)\right)\right. \\
& \left.\cdot f_{2} \chi_{X \backslash 6^{N} B}\right)-m_{\mathrm{Q}} T_{\theta}\left(\left(b_{1}-m_{\widetilde{B}}\left(b_{1}\right)\right)\right. \\
& \left.\cdot f_{1} \chi_{X \backslash 6^{N} B},\left(b_{2}-m_{\widetilde{B}}\left(b_{2}\right)\right) f_{2} \chi_{X \backslash 6^{N} B}\right) \mid \\
& +\mid m_{B} T_{\theta}\left(\left(b_{1}-m_{\tilde{B}}\left(b_{1}\right)\right)\right. \\
& \text { - } \left.f_{1} \chi_{6^{N} B \backslash(6 / 5) B},\left(b_{2}-m_{\widetilde{B}}\left(b_{2}\right)\right) f_{2} \chi_{X \backslash(6 / 5) B}\right) \mid \\
& +\mid m_{B} T_{\theta}\left(\left(b_{1}-m_{\widetilde{B}}\left(b_{1}\right)\right) f_{1} \chi_{X \backslash(6 / 5) B},\left(b_{2}-m_{\widetilde{B}}\left(b_{2}\right)\right)\right. \\
& \left.\cdot f_{2} \chi_{6^{N} B \backslash(6 / 5) B}\right)|+| m_{\mathrm{Q}} T_{\theta}\left(\left(b_{1}-m_{\mathrm{Q}}\left(b_{1}\right)\right)\right. \\
& \text { - } \left.f_{1} \chi_{6^{N} B \backslash(6 / 5) \mathrm{Q}},\left(b_{2}-m_{\mathrm{Q}}\left(b_{2}\right)\right) f_{2} \chi_{X \mid 6^{N} B}\right) \mid \\
& +\mid m_{\mathrm{Q}} T_{\theta}\left(\left(b_{1}-m_{\mathrm{Q}}\left(b_{1}\right)\right)\right. \\
& \text { - } \left.f_{1} \chi_{X \backslash(6 / 5) \mathrm{Q}},\left(b_{2}-m_{\mathrm{Q}}\left(b_{2}\right)\right) f_{2} \chi_{6^{\mathrm{N}} B \backslash(6 / 5) \mathrm{Q}}\right) \mid=: J_{1}+J_{2} \\
& +J_{3}+J_{4}+J_{5}+J_{6} \text {. }
\end{aligned}
$$

Using the method to estimate $I V_{4}$,

$$
J_{1} \leq C\left\|b_{1}\right\|_{*}\left\|b_{2}\right\|_{*} M_{p_{1},(5)} f_{1}(x) M_{p_{2},(5)} f_{2}(x) .
$$

Let us estimate $J_{2}$. As

$$
\begin{aligned}
& T_{\theta}\left(\left(b_{1}-m_{\mathrm{Q}}\left(b_{1}\right)\right) f_{1} \chi_{X \backslash 6^{N} B},\left(b_{2}-m_{\mathrm{Q}}\left(b_{2}\right)\right) f_{2} \chi_{X \backslash 6^{N} B}\right) \\
& \cdot(z)-T_{\theta}\left(\left(b_{1}-m_{\widetilde{B}}\left(b_{1}\right)\right) f_{1} \chi_{X \backslash 6^{N} B},\left(b_{2}-m_{\widetilde{B}}\left(b_{2}\right)\right)\right. \\
& \left.\cdot f_{2} \chi_{X \mid 6^{N} B}\right)(z)=\left(m_{\mathrm{Q}}\left(b_{2}\right)-m_{\widetilde{B}}\left(b_{2}\right)\right) \\
& \cdot T_{\theta}\left(\left(b_{1}-m_{\mathrm{Q}}\left(b_{1}\right)\right) f_{1} \chi_{X \backslash 6^{N} B}, f_{2} \chi_{X \backslash 6^{N} B}\right)(z) \\
& +\left(m_{\mathrm{Q}}\left(b_{1}\right)-m_{\widetilde{B}}\left(b_{1}\right)\right) T_{\theta}\left(f_{1} \chi_{X \mid 6^{N} B},\left(b_{2}-m_{\mathrm{Q}}\left(b_{2}\right)\right)\right. \\
& \left.\cdot f_{2} \chi_{X \mid 6^{N} B}\right)(z)+\left(m_{\mathrm{Q}}\left(b_{1}\right)-m_{\widetilde{B}}\left(b_{1}\right)\right)\left(m_{\mathrm{Q}}\left(b_{2}\right)\right. \\
& \left.-m_{\widetilde{B}}\left(b_{2}\right)\right) T_{\theta}\left(f_{1} \chi_{X \backslash 6^{N} B}, f_{2} \chi_{X \backslash 6^{N} B}\right)(z),
\end{aligned}
$$

then

$$
\begin{aligned}
& J_{2} \leq \mid\left(m_{\mathrm{Q}}\left(b_{2}\right)-m_{\widetilde{B}}\left(b_{2}\right)\right) \frac{1}{\mu(\mathrm{Q})} \\
& \cdot \int_{Q} T_{\theta}\left(\left(b_{1}-m_{\mathrm{Q}}\left(b_{1}\right)\right) f_{1} \chi_{X \backslash 6^{N} B}, f_{2} \chi_{X \backslash 6^{N} B}\right) \\
& \cdot(z) d \mu(z)|+|\left(m_{\mathrm{Q}}\left(b_{1}\right)-m_{\widetilde{B}}\left(b_{1}\right)\right) \frac{1}{\mu(Q)} \\
& \cdot \int_{Q} T_{\theta}\left(\left(f_{1} \chi_{X \backslash 6^{N} B}, b_{2}-m_{\mathrm{Q}}\left(b_{2}\right)\right) f_{2} \chi_{X \backslash 6^{N} B}\right) \\
& \cdot(z) d \mu(z)|+|\left(m_{\mathrm{Q}}\left(b_{1}\right)-m_{\widetilde{B}}\left(b_{1}\right)\right)\left(m_{\mathrm{Q}}\left(b_{2}\right)\right. \\
& \left.-m_{\widetilde{B}}\left(b_{2}\right)\right) \frac{1}{\mu(Q)} \int_{Q} T_{\theta}\left(f_{1} \chi_{X \backslash 6^{N} B}, f_{2} \chi_{X \backslash 6^{N} B}\right)(z) \mid \\
& =: J_{21}+J_{22}+J_{23} \text {. }
\end{aligned}
$$

Let us estimate $J_{21}$. Note that $(6 / 5) Q \subset 6^{N} B$; write

$$
\begin{aligned}
T_{\theta} & \left(\left(b_{1}-m_{\mathrm{Q}}\left(b_{1}\right)\right) f_{1} \chi_{X \backslash 6^{N} B}, f_{2} \chi_{X \backslash 6^{N} B}\right)(z) \\
& =T_{\theta}\left(\left(b_{1}-m_{\mathrm{Q}}\left(b_{1}\right)\right) f_{1}, f_{2}\right)(z) \\
& -T_{\theta}\left(\left(b_{1}-m_{\mathrm{Q}}\left(b_{1}\right)\right) f_{1} \chi_{(6 / 5) \mathrm{Q}}, f_{2} \chi_{(6 / 5) \mathrm{Q}}\right)(z) \\
& -T_{\theta}\left(\left(b_{1}-m_{\mathrm{Q}}\left(b_{1}\right)\right) f_{1} \chi_{X \backslash(6 / 5) \mathrm{Q}}, f_{2} \chi_{6^{N} B}\right)(z) \\
& -T_{\theta}\left(\left(b_{1}-m_{\mathrm{Q}}\left(b_{1}\right)\right) f_{1} \chi_{6^{N} B}, f_{2} \chi_{X \backslash(6 / 5) \mathrm{Q}}\right)(z)
\end{aligned}
$$




$$
\begin{aligned}
& +T_{\theta}\left(\left(b_{1}-m_{\mathrm{Q}}\left(b_{1}\right)\right) f_{1} \chi_{6^{\mathrm{N}} B \backslash(6 / 5) \mathrm{Q}}, f_{2} \chi_{6^{\mathrm{N}} B \backslash(6 / 5) \mathrm{Q}}\right) \\
& \cdot(z)=: E_{1}+E_{2}+E_{3}+E_{4}+E_{5} .
\end{aligned}
$$

Let us estimate $E_{1}$ firstly. Since $Q$ is a doubling ball, we have

$$
\begin{aligned}
& \frac{1}{\mu(Q)} \int_{Q}\left|T_{\theta}\left(\left(b_{1}-b_{1}(z)\right) f_{1}, f_{2}\right)(z)\right| d \mu(z) \\
& \quad \leq C M_{s,(6)}\left(\left[b_{1}, T_{\theta}\right] f_{1}, f_{2}\right)(x) \\
& \frac{1}{\mu(Q)} \int_{Q}\left|\left(b_{1}(z)-m_{Q}\left(b_{1}\right)\right) T_{\theta}\left(f_{1}, f_{2}\right)(z)\right| d \mu(z) \\
& \quad \leq C\left\|b_{1}\right\|_{*} M_{s,(6)}\left(T_{\theta}\left(f_{1}, f_{2}\right)\right)(x) .
\end{aligned}
$$

Thus,

$$
\begin{aligned}
\left|m_{\mathrm{Q}}\left(E_{1}\right)\right| \leq & \left|m_{\mathrm{Q}}\left(T_{\theta}\left(\left(b_{1}-b_{1}(z)\right) f_{1}, f_{2}\right)\right)\right| \\
& +\left|m_{\mathrm{Q}}\left(\left(b_{1}(z)-m_{\mathrm{Q}}\left(b_{1}\right)\right) T_{\theta}\left(f_{1}, f_{2}\right)\right)\right| \\
\leq & C M_{s,(6)}\left(\left[b_{1}, T_{\theta}\right] f_{1}, f_{2}\right)(x) \\
& +\left\|b_{1}\right\|_{*} M_{s,(6)}\left(T_{\theta}\left(f_{1}, f_{2}\right)\right)(x) .
\end{aligned}
$$

For $E_{2}$, let $v>1$ and $1<s_{1}<p_{1}$ such that $1 / v=1 / s_{1}+1 / p_{2}$; denote $1 / s_{1}=1 / s_{2}+1 / p_{1}$. Note that $Q$ is a doubling ball. By Lemmas 16 and 14 and Hölder's inequality,

$$
\begin{aligned}
& \left|m_{\mathrm{Q}}\left(E_{2}\right)\right| \leq C\left\|E_{2}\right\|_{L^{\nu, \infty}(\mathrm{Q}, d \mu(z) / \mu(\mathrm{Q}))} \leq C\left(\frac{1}{\mu(\mathrm{Q})}\right. \\
& \left.\cdot \int_{(6 / 5) \mathrm{Q}}\left|\left(b_{1}(z)-m_{\mathrm{Q}}\left(b_{1}\right)\right) f_{1}(z)\right|^{s_{1}} d \mu(z)\right)^{1 / s_{1}} \\
& \cdot\left(\frac{1}{\mu(Q)} \int_{(6 / 5) \mathrm{Q}}\left|f_{2}(z)\right|^{p_{2}} d \mu(z)\right)^{1 / p_{2}} \\
& \leq C\left(\frac{1}{\mu(6 \mathrm{Q})}\right. \\
& \left.\cdot \int_{(6 / 5) \mathrm{Q}}\left|b_{1}(z)-m_{\mathrm{Q}}\left(b_{1}\right)\right|^{s_{2}} d \mu(z)\right)^{1 / s_{2}}\left(\frac{1}{\mu(6 Q)}\right. \\
& \left.\cdot \int_{(6 / 5) \mathrm{Q}}\left|f_{1}(z)\right|^{p_{1}} d \mu(z)\right)^{1 / p_{1}} \times\left(\frac{1}{\mu(6 Q)}\right. \\
& \left.\cdot \int_{(6 / 5) \mathrm{Q}}\left|f_{2}(z)\right|^{p_{2}} d \mu(z)\right)^{1 / p_{2}} \leq\left\|b_{1}\right\|_{*} M_{p_{1},(5)} f_{1}(x) \\
& \cdot M_{p_{2},(5)} f_{2}(x) .
\end{aligned}
$$

For $E_{3}$, by (i) of Definition 5, Lemma 14, and Hölder's inequality,

$$
\begin{aligned}
& \left|E_{3}\right| \leq C \int_{6^{N} B} \int_{X \backslash(6 / 5) \mathrm{Q}} \frac{\left|b_{1}\left(y_{1}\right)-m_{\mathrm{Q}}\left(b_{1}\right)\right|\left|f_{1}\left(y_{1}\right)\right|\left|f_{2}\left(y_{2}\right)\right| d \mu\left(y_{1}\right) d \mu\left(y_{2}\right)}{\left[\sum_{j=1}^{2} \lambda\left(z, d\left(x, y_{j}\right)\right)\right]^{2}} \leq C \int_{6^{N} B}\left|f_{2}\left(y_{2}\right)\right| d \mu\left(y_{2}\right) \\
& \cdot \sum_{k=1}^{\infty} \int_{6^{k}(6 / 5) \mathrm{Q}} \frac{\left|b_{1}\left(y_{1}\right)-m_{\mathrm{Q}}\left(b_{1}\right)\right|\left|f_{1}\left(y_{1}\right)\right|}{\left(\lambda\left(z, 6^{k-1}(6 / 5) r_{\mathrm{Q}}\right)\right)^{2}} d \mu\left(y_{1}\right) \leq C \int_{6^{N} B}\left|f_{2}\left(y_{2}\right)\right| d \mu\left(y_{2}\right) \sum_{k=1}^{\infty} 6^{-k m} \\
& \times \int_{6^{k}(6 / 5) \mathrm{Q}} \frac{1}{\lambda\left(z,(6 / 5) r_{\mathrm{Q}}\right)} \frac{\left|b_{1}\left(y_{1}\right)-m_{\mathrm{Q}}\left(b_{1}\right)\right|\left|f_{1}\left(y_{1}\right)\right| d \mu\left(y_{1}\right)}{\lambda\left(z, 6^{k-1}(6 / 5) r_{\mathrm{Q}}\right)} \leq C \frac{1}{\lambda\left(z, 6 r_{\mathrm{Q}}\right)} \int_{6^{N} B}\left|f_{2}\left(y_{2}\right)\right| d \mu\left(y_{2}\right) \\
& \cdot \sum_{k=1}^{\infty} 6^{-k m} \frac{1}{\lambda\left(z, 5 \times 6^{k}(6 / 5) r_{\mathrm{Q}}\right)} \times\left[\int_{6^{k}(6 / 5) \mathrm{Q}}\left|b_{1}\left(y_{1}\right)-m_{6^{k}(6 / 5) \mathrm{Q}}\left(b_{1}\right)\right|\left|f_{1}\left(y_{1}\right)\right| d \mu\left(y_{1}\right)\right. \\
& \left.+\int_{\sigma^{k}(6 / 5) \mathrm{Q}}\left|m_{6^{k}(6 / 5) \mathrm{Q}}\left(b_{1}\right)-m_{\mathrm{Q}}\left(b_{1}\right)\right|\left|f_{1}\left(y_{1}\right)\right| d \mu\left(y_{1}\right)\right] \leq C \frac{1}{\lambda\left(z, 6 r_{\mathrm{Q}}\right)} \int_{6^{N} B}\left|f_{2}\left(y_{2}\right)\right| d \mu\left(y_{2}\right) \sum_{k=1}^{\infty} 6^{-k m} \\
& \times\left[\left(\frac{1}{\lambda\left(z, 5 \times 6^{k}(6 / 5) r_{\mathrm{Q}}\right)} \int_{6^{k}(6 / 5) \mathrm{Q}}\left|b_{1}\left(y_{1}\right)-m_{6^{k}(6 / 5) \mathrm{Q}}\left(b_{1}\right)\right|^{p_{1}^{\prime}} d \mu\left(y_{1}\right)\right)^{1 / p_{1}^{\prime}}\right. \\
& \left.\times\left(\frac{1}{\lambda\left(z, 5 \times 6^{k}(6 / 5) r_{\mathrm{Q}}\right)} \int_{6^{k}(6 / 5) \mathrm{Q}}\left|f_{1}\left(y_{1}\right)\right|^{p_{1}} d \mu\left(y_{1}\right)\right)^{1 / p_{1}}+k\left\|b_{1}\right\|_{*} \frac{1}{\lambda\left(z, 5 \times 6^{k}(6 / 5) r_{\mathrm{Q}}\right)} \int_{6^{k}(6 / 5) \mathrm{Q}}\left|f_{1}\left(y_{1}\right)\right| d \mu\left(y_{1}\right)\right] \\
& \leq C \sum_{k=1}^{N} \frac{1}{\lambda\left(z, 6 r_{\mathrm{Q}}\right)} \int_{6^{k} B}\left|f_{2}\left(y_{2}\right)\right| d \mu\left(y_{2}\right)\left\|b_{1}\right\|_{*} M_{p_{1},(5)} f_{1}(x) \leq C \sum_{k=1}^{N} \frac{\mu\left(5 \times 6^{k} B\right)}{\lambda\left(z, 5 \times 6^{k} r_{B}\right)} \frac{\lambda\left(z, 5 \times 6^{k} r_{B}\right)}{\lambda\left(z, 6 r_{\mathrm{Q}}\right)} \times \frac{1}{\mu\left(5 \times 6^{k} B\right)} \\
& \cdot \int_{6^{k} B}\left|f_{2}\left(y_{2}\right)\right| d \mu\left(y_{2}\right)\left\|b_{1}\right\|_{*} M_{p_{1},(5)} f_{1}(x) \leq C K_{B, Q}\left\|b_{1}\right\|_{*} M_{p_{1},(5)} f_{1}(x) M_{p_{2},(5)} f_{2}(x) \text {. }
\end{aligned}
$$


Thus,

$$
\left|m_{\mathrm{Q}}\left(E_{3}\right)\right| \leq C K_{B, \mathrm{Q}}\left\|b_{1}\right\|_{*} M_{p_{1},(5)} f_{1}(x) M_{p_{2},(5)} f_{2}(x) .
$$

Also, we have

$$
\begin{aligned}
& \left|m_{Q}\left(E_{4}\right)\right|+\left|m_{Q}\left(E_{5}\right)\right| \\
& \quad \leq C\left\|b_{1}\right\|_{*} M_{p_{1},(5)} f_{1}(x) M_{p_{2},(5)} f_{2}(x) .
\end{aligned}
$$

By (26) in Lemma 14,

$$
\begin{aligned}
J_{21} & \leq C K_{B, Q}^{2}\left\{\left\|b_{1}\right\|_{*}\left\|b_{2}\right\|_{*} M_{s,(6)}\left(T_{\theta}\left(f_{1}, f_{2}\right)\right)(x)\right. \\
& +\left\|b_{1}\right\|_{*} M_{s,(6)}\left(\left[b_{2}, T_{\theta}\right]\left(f_{1}, f_{2}\right)\right)(x) \\
& +\left\|b_{2}\right\|_{*} M_{s,(6)}\left(\left[b_{1}, T_{\theta}\right]\left(f_{1}, f_{2}\right)\right)(x) \\
& \left.+\left\|b_{1}\right\|_{*}\left\|b_{2}\right\|_{*} M_{p_{1},(5)} f_{1}(x) M_{p_{2},(5)} f_{2}(x)\right\} .
\end{aligned}
$$

$J_{22}$ and $J_{23}$ also have similar estimate of $J_{21}$. Therefore,

$$
\begin{aligned}
J_{2} & \leq C K_{B, Q}^{2}\left\{\left\|b_{1}\right\|_{*}\left\|b_{2}\right\|_{*} M_{s,(6)}\left(T_{\theta}\left(f_{1}, f_{2}\right)\right)(x)\right. \\
& +\left\|b_{1}\right\|_{*} M_{s,(6)}\left(\left[b_{2}, T_{\theta}\right]\left(f_{1}, f_{2}\right)\right)(x) \\
& +\left\|b_{2}\right\|_{*} M_{s,(6)}\left(\left[b_{1}, T_{\theta}\right]\left(f_{1}, f_{2}\right)\right)(x) \\
& \left.+\left\|b_{1}\right\|_{*}\left\|b_{2}\right\|_{*} M_{p_{1},(5)} f_{1}(x) M_{p_{2},(5)} f_{2}(x)\right\} .
\end{aligned}
$$

Using the similar method to estimate $I V_{2}$,

$$
\begin{aligned}
J_{3} & +J_{4}+J_{5}+J_{6} \\
& \leq C\left\|b_{1}\right\|_{*}\left\|b_{2}\right\|_{*} M_{p_{1},(5)} f_{1}(x) M_{p_{2},(5)} f_{2}(x) .
\end{aligned}
$$

Hence, (33) is proved. Thus, the result of Lemma 18 is proved.

Proof of Theorem 10. Because the proof of the result of $\|\mu\|<$ $\infty$ is similar to that of $\|\mu\|=\infty$, now we only prove the result of $\|\mu\|=\infty$. Let $0<\eta<1 / 2,1<p_{1}, p_{2}, q<\infty, 1 / q=$ $1 / p_{1}+1 / p_{2}, 1<s<q, f_{1} \in L^{p_{1}}(\mu), f_{2} \in L^{p_{2}}(\mu)$, and $b_{1}, b_{2} \in$ $\operatorname{RBMO}(\mu)$. By Lemmas 12-14 and 17 and Hölder's inequality, then

$$
\begin{aligned}
& \left\|\left[b_{1}, b_{2}, T_{\theta}\right]\left(f_{1}, f_{2}\right)\right\|_{L^{q}(\mu)} \\
& \leq\left\|N_{\eta}\left(\left[b_{1}, b_{2}, T_{\theta}\right]\left(f_{1}, f_{2}\right)\right)\right\|_{L^{q}(\mu)} \\
& \leq C\left\|M_{\eta}^{\sharp}\left(\left[b_{1}, b_{2}, T_{\theta}\right]\left(f_{1}, f_{2}\right)\right)\right\|_{L^{q}(\mu)} \\
& \leq C\left\|b_{1}\right\|_{*}\left\|b_{2}\right\|_{*}\left\|M_{s,(6)}\left(T_{\theta}\left(f_{1}, f_{2}\right)\right)\right\|_{L^{q}(\mu)} \\
& \quad+C\left\|b_{1}\right\|_{*}\left\|M_{s,(6)}\left(\left[b_{2}, T_{\theta}\right]\left(f_{1}, f_{2}\right)\right)\right\|_{L^{q}(\mu)} \\
& \quad+C\left\|b_{2}\right\|_{*}\left\|M_{s,(6)}\left(\left[b_{1}, T_{\theta}\right]\left(f_{1}, f_{2}\right)\right)\right\|_{L^{q}(\mu)} \\
& \quad+C\left\|b_{1}\right\|_{*}\left\|b_{2}\right\|_{*}\left\|M_{p_{1},(5)}\left(f_{1}\right) M_{p_{2},(5)}\left(f_{2}\right)\right\|_{L^{q}(\mu)}
\end{aligned}
$$

$$
\begin{aligned}
& \leq C\left\|b_{1}\right\|_{*}\left\|b_{2}\right\|_{*}\left\|f_{1}\right\|_{L^{p_{1}}(\mu)}\left\|f_{2}\right\|_{L^{p_{2}}(\mu)} \\
& +C\left\|b_{1}\right\|_{*}\left\|\left(\left[b_{2}, T_{\theta}\right]\left(f_{1}, f_{2}\right)\right)\right\|_{L^{q}(\mu)} \\
& +C\left\|b_{2}\right\|_{*}\left\|\left(\left[b_{1}, T_{\theta}\right]\left(f_{1}, f_{2}\right)\right)\right\|_{L^{q}(\mu)} \\
& \leq C\left\|b_{1}\right\|_{*}\left\|b_{2}\right\|_{*}\left\|f_{1}\right\|_{L^{p_{1}}(\mu)}\left\|f_{2}\right\|_{L^{p_{2}}(\mu)} \\
& +C\left\|b_{1}\right\|_{*}\left\|N_{\eta}\left(\left[b_{2}, T_{\theta}\right]\left(f_{1}, f_{2}\right)\right)\right\|_{L^{q}(\mu)} \\
& +C\left\|b_{2}\right\|_{*}\left\|N_{\eta}\left(\left[b_{1}, T_{\theta}\right]\left(f_{1}, f_{2}\right)\right)\right\|_{L^{q}(\mu)} \\
& \leq C\left\|b_{1}\right\|_{*}\left\|b_{2}\right\|_{*}\left\|f_{1}\right\|_{L^{p_{1}}(\mu)}\left\|f_{2}\right\|_{L^{p_{2}}(\mu)} \\
& +C\left\|b_{1}\right\|_{*}\left\|M_{\eta}^{\sharp}\left(\left[b_{2}, T_{\theta}\right]\left(f_{1}, f_{2}\right)\right)\right\|_{L^{q}(\mu)} \\
& +C\left\|b_{2}\right\|_{*}\left\|M_{\eta}^{\sharp}\left(\left[b_{1}, T_{\theta}\right]\left(f_{1}, f_{2}\right)\right)\right\|_{L^{q}(\mu)} \\
& \leq\left\|b_{1}\right\|_{*}\left\|b_{2}\right\|_{*}\left\|f_{1}\right\|_{L^{p_{1}}(\mu)}\left\|f_{2}\right\|_{L^{p_{2}}(\mu)} \\
& +C\left\|b_{1}\right\|_{*}\left\|M_{s,(6)}\left(T_{\theta}\left(f_{1}, f_{2}\right)\right)\right\|_{L^{q}(\mu)} \\
& +C\left\|b_{1}\right\|_{*}\left\|M_{p_{1},(5)}\left(f_{1}\right) M_{p_{2},(5)}\left(f_{2}\right)\right\|_{L^{q}(\mu)} \\
& +C\left\|b_{2}\right\|_{*}\left\|M_{s,(6)}\left(T_{\theta}\left(f_{1}, f_{2}\right)\right)\right\|_{L^{q}(\mu)} \\
& +C\left\|b_{2}\right\|_{*}\left\|M_{p_{1},(5)}\left(f_{1}\right) M_{p_{2},(5)}\left(f_{2}\right)\right\|_{L^{q}(\mu)} \\
& \leq C\left\|b_{1}\right\|_{*}\left\|b_{2}\right\|_{*}\left\|f_{1}\right\|_{L^{p_{1}}(\mu)}\left\|f_{2}\right\|_{L^{p_{2}}(\mu)} .
\end{aligned}
$$

Thus, the proof of Theorem 10 is finished.

\section{Competing Interests}

The authors declare that they have no competing interests.

\section{Acknowledgments}

This work was supported by Natural Science Foundation of Anhui Province (no. 1608085QA12) and Natural Science Foundation of Education Committee of Anhui Province (nos. KJ2015A117 and KJ2016A506).

\section{References}

[1] T. Hytönen, "A framework for non-homogeneous analysis on metric spaces, and the RBMO space of Tolsa," Publicacions Matemàtiques, vol. 54, no. 2, pp. 485-504, 2010.

[2] T. Hytönen, D. Yang, and D. Yang, "The Hardy space $H^{1}$ on nonhomogeneous metric spaces," Mathematical Proceedings of the Cambridge Philosophical Society, vol. 153, no. 1, pp. 9-31, 2012.

[3] T. A. Bui and X. T. Duong, "Hardy spaces, regularized BMO spaces and the boundedness of Calderón-Zygmund operators on non-homogeneous spaces," Journal of Geometric Analysis, vol. 23, no. 2, pp. 895-932, 2013.

[4] S. Liu, D. Yang, and D. Yang, "Boundedness of CalderónZygmund operators on non-homogeneous metric measure 
spaces: equivalent characterizations," Journal of Mathematical Analysis and Applications, vol. 386, no. 1, pp. 258-272, 2012.

[5] X. Fu, D. Yang, and W. Yuan, "Boundedness of multilinear commutators of Calderón-Zygmund operators on Orlicz spaces over non-homogeneous spaces," Taiwanese Journal of Mathematics, vol. 16, no. 6, pp. 2203-2238, 2012.

[6] X. Fu, D. Yang, and W. Yuan, "Generalized fractional integrals and their commutators over non-homogeneous metric measure spaces," Taiwanese Journal of Mathematics, vol. 18, no. 2, pp. 509-557, 2014.

[7] C. Yonghui and Z. Jiang, “The boundedness of Marcinkiewicz integrals commutators on non-homogeneous metric measure spaces," Journal of Inequalities and Applications, vol. 2015, article no. 259, 2015.

[8] X. Fu, H. Lin, D. Yang, and D. Yang, "Hardy spaces $H^{p}$ over nonhomogeneous metric measure spaces and their applications," Science China Mathematics, vol. 58, no. 2, pp. 309-388, 2015.

[9] G. Hu, Y. Meng, and D. Yang, "Weighted norm inequalities for multilinear Calderón-Zygmund operators on nonhomogeneous metric measure spaces," Forum Mathematicum, vol. 26, no. 5, pp. 1289-1322, 2014.

[10] T. Hytönen and H. Martikainen, "Non-homogeneous Tb theorem and random dyadic cubes on metric measure spaces," Journal of Geometric Analysis, vol. 22, no. 4, pp. 1071-1107, 2012.

[11] T. Hytönen, S. Liu, D. Yang, and D. Yang, "Boundedness of Calderón-Zygmund operators on non-homogeneous metric measure spaces," Canadian Journal of Mathematics, vol. 64, no. 4, pp. 892-923, 2012.

[12] H. Lin, S. Wu, and D. Yang, "Boundedness of certain commutators over non-homogeneous metric measure spaces," Analysis and Mathematical Physics, 2016.

[13] S. Liu, Y. Meng, and D. Yang, "Boundedness of maximal Calderón-Zygmund operators on non-homogeneous metric measure spaces," Proceedings of the Royal Society of Edinburgh Section A: Mathematics, vol. 144, no. 3, pp. 567-589, 2014.

[14] C. Tan and J. Li, "Littlewood-Paley theory on metric spaces with non doubling measures and its applications," Science China Mathematics, vol. 58, no. 5, pp. 983-1004, 2015.

[15] R. Xie, H. Gong, and X. Zhou, "Commutators of multilinear singular integral operators on non-homogeneous metric measure spaces," Taiwanese Journal of Mathematics, vol. 19, no. 3, pp. 703-723, 2015.

[16] K. Yabuta, "Generalization of Calderón-Zygmund operators," Studia Mathematica, vol. 82, pp. 17-31, 1985.

[17] R. Xie and L. Shu, “ $\Theta$-type Calderón-Zygmund operators with non-doubling measures," Acta Mathematicae Applicatae Sinica, English Series, vol. 29, no. 2, pp. 263-280, 2013.

[18] C. Ri and Z. Zhang, "Boundedness of $\theta$-type CalderónZygmund operators on Hardy spaces with non-doubling measures," Journal of Inequalities and Applications, vol. 323, pp. 1-10, 2015.

[19] C. Ri and Z. Zhang, "Boundedness of $\theta$-type CalderónZygmund operators on nonhomogeneous metric measure spaces," Frontiers of Mathematics in China, vol. 11, no. 1, pp. 141153, 2016.

[20] T. Zheng, X. Tao, and X. Wu, "Bilinear Calderón-Zygmund operators of type $\omega(\mathrm{t})$ on non-homogeneous space," Journal of Inequalities and Applications, vol. article 113, pp. 1-18, 2014.

[21] T. Zheng, Z. Wang, and W. Xiao, "Maximal bilinear CalderónZygmund operators of type $\omega(\mathrm{t})$ on non-homogeneous space," Annals of Functional Analysis, vol. 6, no. 4, pp. 134-154, 2015.
[22] H. Gong, R. Xie, and C. Xu, "Multilinear fractional integral operators on non-homogeneous metric measure spaces," Journal of Inequalities and Applications, vol. 2016, article no. 275, 2016.

[23] H. Lin and D. Yang, "Spaces of type BLO on non-homogeneous metric measure," Frontiers of Mathematics in China, vol. 6, no. 2, pp. 271-292, 2011.

[24] X. Tolsa, "BMO, $\mathrm{H}^{1}$, and Calderón-zygmund operators for non doubling measures," Mathematische Annalen, vol. 319, pp. 89101, 2001. 


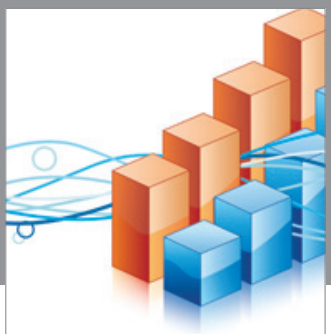

Advances in

Operations Research

vatem alat4

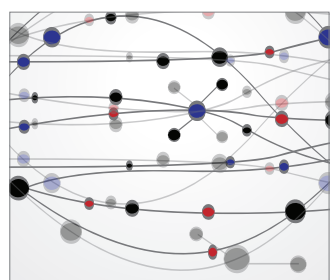

\section{The Scientific} World Journal
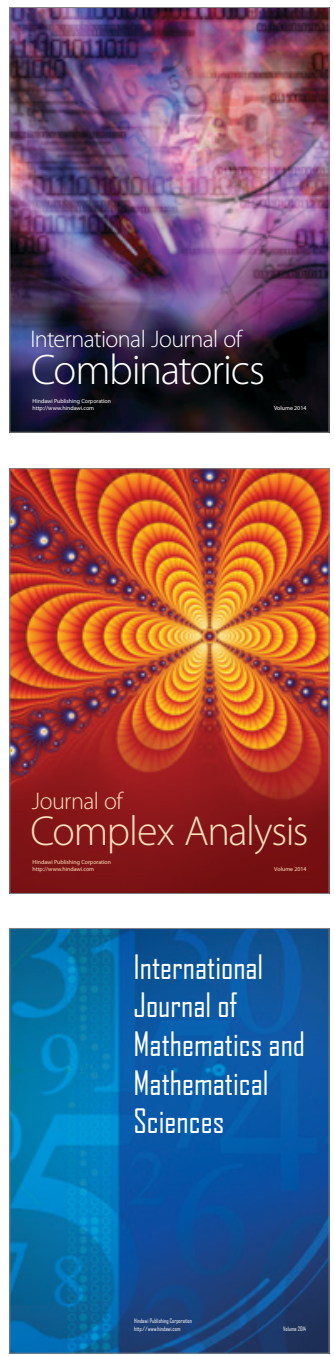
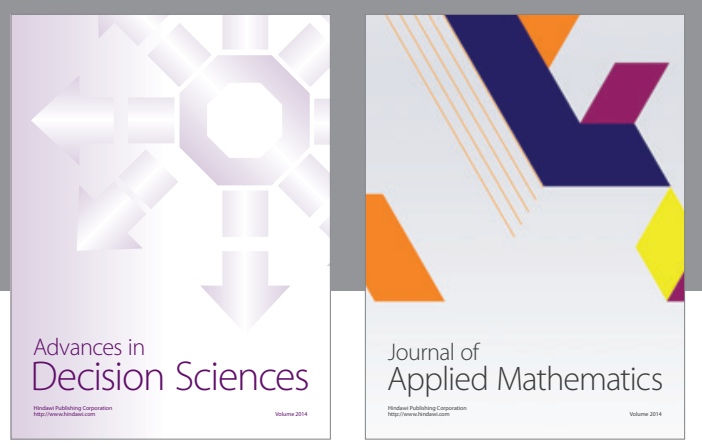

Algebra

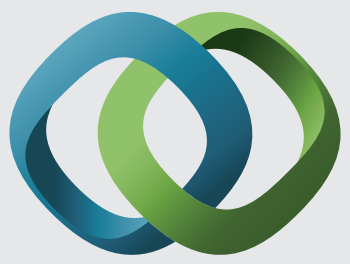

\section{Hindawi}

Submit your manuscripts at

https://www.hindawi.com
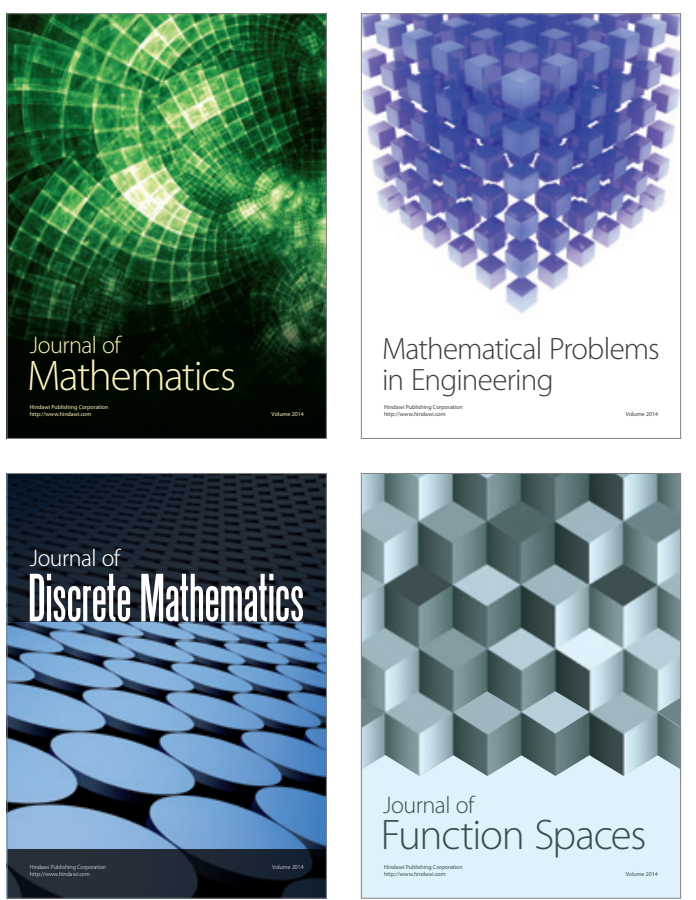

Mathematical Problems in Engineering
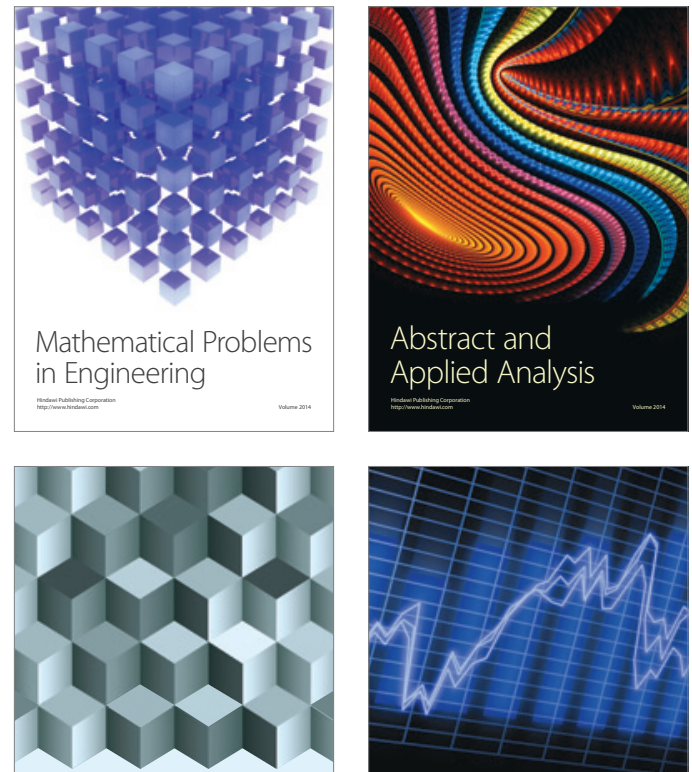

Journal of

Function Spaces

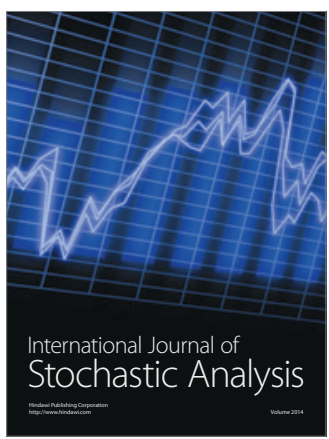

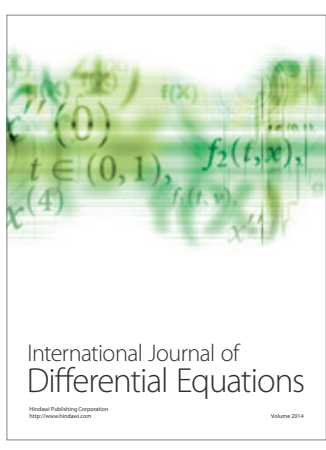
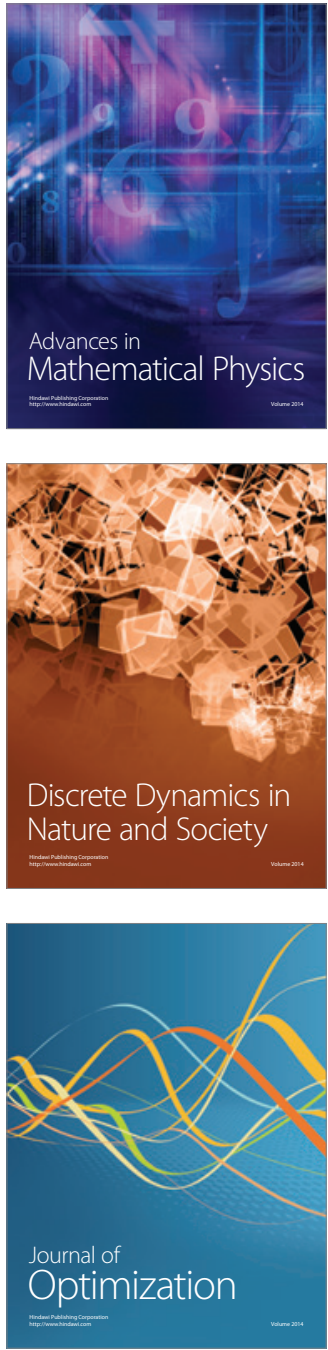